\title{
The effect of makeup behavior and makeup perception on makeup usage level before and during the COVID-19 pandemic
}

\author{
Sun-Hyoung Kim, $\mathrm{PhD}^{1}$ (D), Eui-Hyoung Kim, $\mathrm{PhD}^{2}$ (iD \\ 'Department of Beauty Science, Kwangju Women's University, Gwangju, Rep. of Korea \\ ${ }^{2}$ Department of Beauty, Seowon University, Cheongju, Rep. of Korea
}

Background: This study investigated the impact of perceptions on makeup behavior and levels of makeup use before and during the COVID-19 pandemic.

Objective: It is presumed that during the COVID-19 outbreak, due to the restrictions on meeting people, mandatory wearing of facial masks, and the spread of contactless culture, there may have occurred a change in the makeup behavior, makeup perception, and level of makeup use. However, no empirical study has been conducted on this topic. Hence, this study aimed to identify the effect of the COVID-19 pandemic on makeup use in female adults, using an empirical analysis method.

Methods: A survey was conducted on 245 female adults aged 18 years or older, residing in the Gwangju Metropolitan City. Empirical analysis was performed at a significance level of $p<0.05$, using the SPSS WIN 21.0 program. To identify general characteristics of survey respondents, a chi-squared test was performed, and to verify the feasibility of estimation tools, a factor analysis was conducted. To measure the level of reliability, Cronbach's $\alpha$ coefficient was calculated. A paired samples t-test was used to determine the level of makeup use before and during the COVID-19 pandemic. Lastly, a multiple linear regression analysis identified the impact of makeup behavior and makeup perception on the makeup usage level before and during COVID-19.

Results: The analysis of difference in makeup usage before and after the pandemic began showed that makeup usage during the pandemic (mean=2.84) decreased from the pre-pandemic level (mean=3.59). It was also found that higher aesthetics, synchronism, and courtesy as subfactors of makeup behavior led to an increase in makeup usage during the pandemic. In addition, this study also confirmed that respondents with higher social achievement as a subfactor of makeup perception increased their makeup usage during the pandemic.

Conclusion: Overall, makeup usage during COVID-19 decreased compared to the pre-pandemic level, but respondents with higher aesthetics, synchronism, and courtesy, which are subfactors of makeup behavior, and those with higher social achievement, a subfactor of makeup perception, tended to wear more makeup after the pandemic began. These findings may be used as basic data for cosmetic marketing.

Keywords: factors of makeup behavior; makeup perception; makeup usage level 


\section{Introduction}

The desire for beauty is rooted in human instincts, and particularly for female, beauty is said to be a lifelong pursuit [1]. Makeup, one of the means of expressing one's beauty, improves appearance safely at a relatively small cost of money and time, and seems to bear more significance than just decorating one's face [2]. Makeup is a tool to smoothen social life and often brings about a change in self-perception and attitude toward life. However, restrictions on meeting people, mandatory wearing of facial masks, and the spread of contactless culture amid the COVID-19 pandemic have affected makeup usage. Instead of applying makeup on the whole face, people only wear eye makeup, as it is visible even when wearing a mask. Moreover, due to concerns over contact dermatitis caused by prolonged use of masks, consumers are interested in skin care with a different purpose than before. Hence, an empirical study on the comparison of the level of makeup usage in female before and during the COVID-19 pandemic and the impact of the pandemic on makeup behavior is called for, but such studies are nonexistent as of now, except those on the relationship between COVID-19-related mask wearing and makeup satisfaction [3] and those on females' interest in cosmetics and the usage of cosmetics before and during the pandemic [4]. Accordingly, this a study aims to analyze makeup behavior before and during the outbreak and the impact of makeup behavior and makeup perception on the level of makeup usage, which may offer useful basic data for cosmetics marketers.

\section{Materials and methods}

\section{Research subjects and data collection}

The study sample consisted of female adults aged 18 years or older residing in the Gwangju Metropolitan City, who have a right to choose what to wear on their faces and are engaged in social and economic activities. A survey was conducted for two weeks, from the first week to the second week of March 2021. To observe COVID-19 quarantine rules, the purpose of the study was explained to survey participants, seeking their consent via emails, and then the survey was conducted over the Internet.

Profiles of respondents are listed in Table 1. As for age distribution, 26 respondents were aged 18 or 19 years $(10.6 \%)$, 88 were in their 20s (35.9\%), 46 in their 30s (18.8\%), 52 in their 40 s $(21.2 \%), 21$ in their 50 s $(8.6 \%)$, and 12 in their 60 s $(4.9 \%)$, with those in their 20s and 30s making up the largest share. With regard to occupation, there were 78 students (31.8\%), 60 professionals (24.5\%), 43 full-time homemakers (17.6\%), 32 company employees (13.1\%), 18 freelancers $(7.3 \%)$, and 14 civil servants (5.7\%). As for education level, 110 survey respondents were attending or had graduated from universities (including vocational colleges) (44.9\%), 91 graduated from high school or lower (37.1\%), and 44 had completed graduate school or higher (18.0\%). In terms of monthly income, 88 respondents earned less than Korean won (KRW) 2 million (35.9\%), 60 earned KRW 2 to 3 million (24.5\%), 46 earned KRW 4 million or more (18.8\%), and 27 earned from KRW 3 million to less than 4 million (11.0\%). As for marital status, 129 respondents were single (52.7\%) while 116 were married $(47.3 \%)$.

Table 1. General characteristics of survey respondents

\begin{tabular}{|c|c|c|}
\hline Category & Frequency (n) & Share (\%) \\
\hline \multicolumn{3}{|l|}{ Age group (yr) } \\
\hline $18-19$ & 26 & 10.6 \\
\hline $20 \mathrm{~s}$ & 88 & 35.9 \\
\hline $30 \mathrm{~s}$ & 46 & 18.8 \\
\hline $40 \mathrm{~s}$ & 52 & 21.2 \\
\hline $50 \mathrm{~s}$ & 21 & 8.6 \\
\hline $60 \mathrm{~s}$ & 12 & 4.9 \\
\hline \multicolumn{3}{|l|}{ Occupation } \\
\hline Students & 78 & 31.8 \\
\hline Company employees & 32 & 13.1 \\
\hline Civil servants & 14 & 5.7 \\
\hline Professionals & 60 & 24.5 \\
\hline Full-time housewives & 43 & 17.6 \\
\hline Freelancers & 18 & 7.3 \\
\hline \multicolumn{3}{|l|}{ Educational level } \\
\hline Graduate of high school or lower & 91 & 37.1 \\
\hline $\begin{array}{l}\text { Attending or graduated from } \\
\text { universities (including vocational } \\
\text { colleges) }\end{array}$ & 110 & 44.9 \\
\hline $\begin{array}{l}\text { Graduate of graduate schools or } \\
\text { higher }\end{array}$ & 44 & 18.0 \\
\hline \multicolumn{3}{|l|}{ Monthly income (KRW) } \\
\hline Less than 2 million & 88 & 35.9 \\
\hline 2 million to less than 3 million & 60 & 24.5 \\
\hline 3 million to less than 4 million & 27 & 11.0 \\
\hline Exceeding 4 million & 46 & 18.8 \\
\hline Others & 24 & 9.8 \\
\hline \multicolumn{3}{|l|}{ Marital status } \\
\hline Single & 129 & 52.7 \\
\hline Married & 116 & 47.3 \\
\hline Total & 245 & 100 \\
\hline
\end{tabular}

KRW, Korean won. 


\section{Research tools and analysis methodology}

Based on previous studies, this study composed 19 questions on makeup behavior and 6 questions on makeup perception [5-7], and 14 questions about the makeup usage level were selected through content verification of three makeup specialists. The survey consisted of 44 questions including 5 questions regarding general characteristics of respondents. All questions were in the form of five-point Likert scale survey questions.

Empirical analysis was made at a statistical significance level of $\mathrm{p}<0.05$, using the IBM SPSS WIN 21.0 program (IBM Corp., Armonk, NY, USA). To investigate general characteristics of survey respondents, a chi-squared test was conducted, and to measure the feasibility of estimation tools, a factor analysis was used. To verify reliability, Cronbach's $\alpha$ coefficient was calculated. Furthermore, to check if there was a difference in the makeup usage level before and during the COVID-19 pandemic, a paired samples t-test was made. To identify the effect of makeup behavior and makeup perception on the makeup usage level before and during COVID-19, a multiple linear regression analysis was performed.

\section{Results}

\section{Difference in the makeup usage level before and during the COVID-19 pandemic}

A factor analysis was conducted to verify the feasibility of estimation tools used in this study, and Cronbach's $\alpha$ coefficient was estimated to test reliability. Results are as follows. First, tests of the feasibility and reliability of makeup behavior were based on five factors with the coefficient of determination at $80.590 \%$. Factor 1 (19.802\%) was "aesthetics," Factor 2 (18.068\%) was "fashion," Factor 3 (18.029\%) was "interest," Factor 4 (16.737\%) was "synchronism," and Factor 5 (7.955\%) was "courtesy." Second, the feasibility and reliability of makeup perception were tested based on three factors with the coefficient of determination of $80.590 \%$. Factor 1 (30.246\%) was "appearance management," Factor 2 (30.109\%) was "self-satisfaction," and Factor 3 $(27.390 \%)$ was "social achievement." With the factor loading of these three factors being over 0.40 and reliability over 0.60 , the feasibility and reliability of makeup perception were secured. Third, tests of feasibility and reliability of the makeup usage level before and during COVID-19 were based on one factor with the coefficients of determination at $65.592 \%$ and $64.190 \%$, respectively. Subfactors were the makeup usage level before COVID-19 and the makeup usage level during COVID-19 respectively, with all factor loadings of over 0.40 and reliability of
Table 2. Difference in the makeup usage level before and during COVID-19

\begin{tabular}{lccr}
\hline \multicolumn{1}{c}{ Question } & $\begin{array}{c}\text { Before } \\
\text { COVID-19 }\end{array}$ & $\begin{array}{c}\text { During } \\
\text { COVID-19 }\end{array}$ & \multicolumn{1}{c}{ t-value } \\
\hline Basic makeup & $4.48(0.871)$ & $3.96(1.157)$ & $7.965^{* * *}$ \\
Skin makeup & $3.77(1.257)$ & $2.98(1.231)$ & $11.848^{* * *}$ \\
Eyebrow makeup & $3.91(1.291)$ & $3.32(1.372)$ & $8.390^{* * *}$ \\
Eye makeup & $3.39(1.412)$ & $2.93(1.418)$ & $7.064^{* * *}$ \\
Lip makeup & $3.98(1.134)$ & $2.67(1.259)$ & $16.689^{* * *}$ \\
Cheek makeup & $2.96(1.431)$ & $2.13(1.160)$ & $11.106^{* * *}$ \\
Contouring makeup & $2.67(1.434)$ & $1.87(1.085)$ & $10.320^{* * *}$ \\
Total & $3.59(1.026)$ & $2.84(0.997)$ & $16.815^{* * *}$ \\
\hline
\end{tabular}

Values are presented as mean (SD).

***Statistically significant $(\mathrm{p}<0.001)$.

over 0.60 , securing both feasibility and reliability.

To investigate any difference in the makeup usage level before and during the pandemic, a paired samples t-test was conducted with results shown in Table 2. Analysis results found that there was a statistically significant difference before and during the pandemic in terms of the general makeup usage level as well as detailed subcategories including basic makeup, skin makeup, eyebrow makeup, eye makeup, lip makeup, cheek makeup, and contouring makeup $(\mathrm{p}<0.001)$. These results are consistent with the findings of previous studies [8] that the makeup usage level decreased after people began wearing masks during the pandemic.

The general makeup usage level during the pandemic (mean=2.84) decreased from the pre-pandemic level (mean=3.59), and the basic makeup, skin makeup, eyebrow makeup, eye makeup, lip makeup, cheek makeup, and contouring makeup usage levels dropped during the pandemic. That is, the makeup usage level has overall decreased since the outbreak. These results are related to findings of previous studies [3] that in the relationship between the makeup usage level and makeup satisfaction during COVID-19, the voluntary targetoriented attitude has a moderating effect. Moreover, the results are also in line with news reports that the makeup focus of both beauty influencers and the public has shifted from lip makeup before the pandemic to eye makeup during the pandemic, leading to an increase in sales of the relevant products [9].

The impact of makeup behavior on the makeup usage level before and during COVID-19

To measure the impact of makeup behavior on the makeup usage level before and during COVID-19, a multiple linear regression analysis was conducted and results are shown in Table 
Table 3. The impact of makeup behavior on makeup usage level before and during COVID-19

\begin{tabular}{|c|c|c|c|c|c|c|c|c|}
\hline \multirow[t]{2}{*}{ Dependent variable } & \multirow{2}{*}{$\begin{array}{c}\text { Independent } \\
\text { variable }\end{array}$} & \multicolumn{2}{|c|}{$\begin{array}{l}\text { Unstandardized } \\
\text { coefficient }\end{array}$} & \multirow{2}{*}{$\begin{array}{c}\begin{array}{c}\text { Standardized } \\
\text { coefficient }\end{array} \\
\beta\end{array}$} & \multirow[t]{2}{*}{ t-value } & \multirow[t]{2}{*}{ p-value } & \multirow[t]{2}{*}{$\mathbf{F}$} & \multirow{2}{*}{$\begin{array}{c}\mathbf{R}^{2} \\
\left(\text { adjusted } R^{2} \text { ) }\right.\end{array}$} \\
\hline & & B & SE & & & & & \\
\hline \multirow[t]{6}{*}{ Makeup usage level before COVID-19 } & (constant) & 0.663 & 0.274 & & 2.423 & 0.016 & $30.606^{* * *}$ & $0.390(0.378)$ \\
\hline & Aesthetics & 0.500 & 0.084 & 0.459 & $5.929 * * *$ & $<0.001$ & & \\
\hline & Fashion & -0.008 & 0.064 & -0.008 & -0.120 & 0.905 & & \\
\hline & Interest & 0.095 & 0.077 & 0.093 & 1.226 & 0.221 & & \\
\hline & Synchronism & 0.138 & 0.064 & 0.147 & $2.158^{*}$ & 0.032 & & \\
\hline & Courtesy & 0.092 & 0.053 & 0.089 & 1.740 & 0.083 & & \\
\hline \multirow[t]{6}{*}{ Makeup usage level during COVID-19 } & (constant) & -0.078 & 0.269 & & -0.290 & 0.772 & $29.013^{* * *}$ & $0.378(0.365)$ \\
\hline & Aesthetics & 0.431 & 0.083 & 0.407 & $5.209 * * *$ & $<0.001$ & & \\
\hline & Fashion & 0.034 & 0.063 & 0.038 & 0.532 & 0.595 & & \\
\hline & Interest & -0.031 & 0.076 & -0.031 & -0.411 & 0.681 & & \\
\hline & Synchronism & 0.212 & 0.063 & 0.233 & $3.371^{* *}$ & 0.001 & & \\
\hline & Courtesy & 0.200 & 0.052 & 0.199 & $3.846^{* * *}$ & $<0.001$ & & \\
\hline
\end{tabular}

SE, standard error.

Statistically significant $\left({ }^{*} \mathrm{p}<0.05,{ }^{* *} \mathrm{p}<0.01,{ }^{* * *} \mathrm{p}<0.001\right)$.

3. First, the regression about the makeup usage level before COVID-19 was statistically significant with the coefficient of determination standing at $39.0 \%(\mathrm{~F}=30.606, \mathrm{p}<0.001)$. As for independent variables, aesthetics $(\beta=0.459, \mathrm{p}<0.001)$, and synchronism $(\beta=0.147, p<0.05)$ were found to be related to the makeup usage level before COVID-19 with a statistical significance. That is, an increase in aesthetics and synchronism as subfactors of makeup behavior led to a higher makeup usage level before the pandemic, which is consistent with previous studies [1] that found that interest and aesthetics as subfactors of makeup behavior were significant among female college students to boost their psychological well-being. That suggests that makeup behavior is a tool used to satisfy their desire for beauty and results in a smooth and effective social life.

The regression equation of the makeup usage level during the pandemic was statistically significant with the coefficient of determination at $37.8 \%(\mathrm{~F}=29.013, \mathrm{p}<0.001)$. As for independent variables, aesthetics $(\beta=0.407, p<0.001)$, synchronism $(\beta=0.233$, $\mathrm{p}<0.01)$, and courtesy $(\beta=0.199, \mathrm{p}<0.001)$ were found to be related to the makeup usage level during the pandemic with a statistical significance. That is, higher aesthetics, synchronism, and courtesy as subfactors of makeup behavior seem to increase the makeup usage level during the pandemic, because the makeup usage level is related to personal tastes of individuals or the characteristics of occupation, as an essential element for interpersonal contacts in the society. This result is in line with the findings of previous studies [4] that due to mask wearing during the pandemic, the focus of makeup has shifted from makeup of the whole face to eye or eyebrow makeup. It is also related to previous studies [10], which reported that in the relationship between face consciousness and appearance concern, appearance perception has a mediating effect.

The impact of makeup perception on makeup usage level before and during COVID-19

A multiple linear regression analysis was conducted to identify the impact of makeup perception on the makeup usage level before and during COVID-19 and its results are shown in Table 4. First, the coefficient of determination of the regression about the makeup usage level before COVID-19 was $21.6 \%$ and the regression equation was statistically significant $(\mathrm{F}=22.070$, $\mathrm{p}<0.001)$. As for independent variables, social achievement $(\beta=0.321, p<0.001)$ and appearance management $(\beta=0.241$, $\mathrm{p}<0.01$ ) were found to be related to makeup usage level before the pandemic with a statistical significance. In other words, higher social achievement and appearance management as subfactors of makeup perception led to an elevated makeup usage level before the pandemic. This result is consistent with previous study findings [9] that the voluntary target-oriented makeup attitude affected appearance management, which in turn affected appearance satisfaction.

The coefficient of determination of the regression equation about the makeup usage level during COVID-19 was $17.2 \%$ and the regression was statistically significant $(\mathrm{F}=16.721, \mathrm{p}<0.001)$. As for independent variables, social achievement $(\beta=0.316$, $\mathrm{p}<0.001$ ) was found to influence makeup usage level during COVID-19 with a statistical significance. This suggests that a rise in social achievement as a subfactor of makeup perception 
Table 4. The impact of makeup perception on makeup usage level before and during COVID-19

\begin{tabular}{|c|c|c|c|c|c|c|c|c|}
\hline \multirow[t]{2}{*}{ Dependent variable } & \multirow{2}{*}{$\begin{array}{l}\text { Independent } \\
\text { variable }\end{array}$} & \multicolumn{2}{|c|}{$\begin{array}{c}\text { Unstandardized } \\
\text { coefficient }\end{array}$} & \multirow{2}{*}{$\begin{array}{c}\begin{array}{c}\text { Standardized } \\
\text { coefficient }\end{array} \\
\beta \beta\end{array}$} & \multirow{2}{*}{$\begin{array}{l}\text { Dependent } \\
\text { variable }\end{array}$} & \multirow[t]{2}{*}{ p-value } & \multirow[t]{2}{*}{$\mathbf{F}$} & \multirow{2}{*}{$\begin{array}{c}\mathbf{R}^{2} \\
\left(\text { adjusted } R^{2} \text { ) }\right.\end{array}$} \\
\hline & & B & SE & & & & & \\
\hline \multirow[t]{4}{*}{ Makeup usage level before COVID-19 } & (Constant) & 0.217 & 0.426 & & 0.510 & 0.610 & $22.070 * * *$ & $0.216(0.206)$ \\
\hline & $\begin{array}{l}\text { Appearance } \\
\text { management }\end{array}$ & 0.322 & 0.092 & 0.241 & $3.508^{* *}$ & 0.001 & & \\
\hline & Self-satisfaction & -0.016 & 0.091 & -0.013 & -0.175 & 0.861 & & \\
\hline & Social achievement & 0.468 & 0.097 & 0.321 & $4.815^{* * *}$ & $<0.001$ & & \\
\hline \multirow[t]{4}{*}{ Makeup usage level during COVID-19 } & (Constant) & -0.090 & 0.425 & & -0.212 & 0.832 & $16.721^{* * *}$ & $0.172(0.162)$ \\
\hline & $\begin{array}{l}\text { Appearance } \\
\text { management }\end{array}$ & 0.167 & 0.091 & 0.128 & 1.822 & 0.070 & & \\
\hline & Self-satisfaction & 0.057 & 0.090 & 0.047 & 0.635 & 0.526 & & \\
\hline & Social achievement & 0.448 & 0.097 & 0.316 & $4.620^{* * *}$ & $<0.001$ & & \\
\hline
\end{tabular}

SE, standard error.

Statistically significant $\left({ }^{* *} \mathrm{p}<0.01,{ }^{* * *} \mathrm{p}<0.001\right)$.

led to a higher makeup usage level during the pandemic. This result contradicts previous study findings [10] that decreased makeup behavior during the pandemic is attributed to self-satisfaction, rather than concern about what others think. If people use makeup to satisfy themselves, makeup behavior affects the self-satisfaction level. However, the makeup usage level has decreased after the pandemic began.

\section{Discussion}

Makeup has been practiced as a way to satisfy one's desire for beauty and to express oneself. However, during the COVID-19 outbreak, restrictions on meeting people, mandatory facial mask wearing, and the spread of contactless culture brought about an overall change in makeup usage. This study conducted an empirical investigation of the impact of makeup behavior and perception on the makeup usage level before and during the pandemic. Overall, the makeup usage level has dropped since the pandemic, and a higher level of aesthetics, synchronism, and courtesy, which are subfactors of makeup behavior, and a higher level of social achievement, a subfactor of makeup perception, led to higher level of makeup usage during the pandemic. This result can be attributed to the increased percentage of female who are economically active, and the rise of females' educational attainment. Results of this paper are expected to be used as basic data for cosmetics marketers. However, the author acknowledges that it is not possible to generalize the results of this study as it focused on female adults residing in the Gwangju Metropolitan City only.

\section{Conflicts of interest}

The authors have nothing to disclose.

\section{References}

1. Kim EH, Kim SH. The effects of makeup behavior factors on self-esteem and psychological wellbeing in university Women. J Korean Soc Cosmetol 2019;25:702-12.

2. Kim YE, Kim JD. A study on the use of color cosmetics according to women's appearance interest. J Korean Soc Cosmet Cosmetol 2020;10:289-305.

3. Kim SY, LI SH. Correlation among wearing masks because of COVID-19, makeup satisfaction and goal-oriented attitude. J Converg Inf Technol 2020;12:156-165.

4. Park YM, Kim HY. A study on changes in women's makeup interest and use patterns before and after the outbreak of COVID-19. Asian J Beauty Cosmetol 2021;19:129-38.

5. Choi SK, Gil MC, Kim MS. The effect of state of makeup and makeup behavior factors on self-esteem and interpersonal relationship among middle and high school girls. AJMAHS 2017;7:409-20.

6. Han SJ. The influence of personal color perception, makeup behavior, cosmetic purchase behavior on image management behaviors [dissertation]. Gwangju: Honam University; 2017.

7. Gwak JS. Make-up recognition and behavior according to ego identity and subjective age of middle-aged women [dissertation]. Busan: Tongmyong University; 2014.

8. Lee JA. "Mask beauty" in the corona era [Internet]. Seoul: Cosmetic Mania News; c2021 [cited 2021 Feb 26]. Available from: http://www.cmn.co.kr/sub/news/news_view.asp?news_ 
idx $=35282$.

9. Jo MJ. The effects of the body images and sociocultural attitudes towards appearance of sports participants on beauty management behavior - focused on the levels -[dissertation]. Seoul: SeoKyeong University; 2020.
10. Kim HS. The effects of appearance management behavior and appearance satisfaction on quality of life: socio-cultural and goal-oriented behavioral approach [dissertation]. Gwangju: Chosun University; 2017. 